A. K. Dupree and A. O. Benz, Eds.

\title{
Multi-Wavelength Observations of EV Lacertae
}

\author{
Rachel A. Osten \\ Jansky Fellow, National Radio Astronomy Observatory, \\ Charlottesville, VA 22903, USA
}

Suzanne L. Hawley, Joel Allred

University of Washington, Seattle, WA, USA

Christopher M. Johns-Krull

Rice University, Houston, TX, USA

Christine Roark

University of Iowa, Ames, IA, USA

Carol Ambruster

Villanova University, Villanova, PA, USA

\author{
Alexander Brown, Thomas R. Ayres, Jeffrey L. Linsky \\ University of Colorado, Boulder, CO, USA
}

\begin{abstract}
We report on a large, multi-wavelength campaign to observe variability across the electromagnetic spectrum in the $\mathrm{M}$ dwarf flare star EV Lacertae, in 2001 September. The campaign involved X-ray (Chandra ACIS-S+HETG), UV (HST/STIS), and optical (McDonald) spectra, as well as optical photometry and multi-frequency radio (VLA) observations. EV Lac demonstrated both frequent and extreme variability during the course of the two day intensive recordings. Dispersed X-ray spectra confirm the metal underabundance seen in other active stars. The increase in continuum fluxes at short X-ray wavelengths during flare intervals compared to quiescent intervals signals the creation of high temperature plasma, a signature of the flare process. Multi-wavelength comparisons reveal interesting trends: X-ray flare frequencies are within the range predicted by optical observations, yet there is no correspondence between X-ray flares and optical flares in our data. Two UV flares occur during the rise stages of X-ray flares; a major radio flare is accompanied by a large optical flare, which has no apparent counterpart in the X-ray. The results give conflicting evidence for the applicability of the Neupert effect interpretation in stellar coronae.
\end{abstract}




\section{Introduction}

The study of extreme stellar coronae, such as on $\mathrm{M}$ dwarf flare stars, allows an investigation into regimes of temperature, density, and activity not available from spatially detailed studies of our low-activity Sun. dMe stars are small, at the edge of being considered fully convective, and have intense magnetic fields covering a sizable fraction of the stellar disk. Despite their small size, dMe flare stars produce close to the maximum amount of coronal emission any star seems able to maintain - even in the quiescent state. Flares are fundamentally linked to magnetic processes occurring in their outer atmospheres and involve the entire atmosphere and produce emission all across the electromagnetic spectrum.

EV Lac (dM4.5e) is a young disk population star at a distance of $5.1 \mathrm{pc}$. Its low mass $\left(0.35 \mathrm{M}_{\odot}\right)$ puts it near the dividing line for fully convective stars (Delfosse et al. 1998). EV Lac has very strong $4 \mathrm{kG}$ magnetic fields covering $>50 \%$ of the stellar surface (Johns-Krull \& Valenti 1996; Saar 1994) so coronal phenomena are dominated by magnetic field interactions. The optical variability of EV Lac is well known; Kodaira, Ichimura, \& Nishimura (1976) saw a flare on EV Lac with an increase of 5.9 magnitudes in U band. Leto et al. (1997)'s study of optical variability determined a B band flaring rate of $0.16-0.37$ flares $\mathrm{hr}^{-1}$. The star is variable at radio wavelengths as well: White, Jackson, \& Kundu (1989) determined an upper limit of $0.3 \mathrm{mJy}$ at $6 \mathrm{~cm}$, while Caillault, Drake, \& Florkowski (1988) detected the source at $1.0 \mathrm{mJy}$. At X-ray wavelengths, EV Lac has shown dramatically different types of flares: from extremely short duration events that last a few minutes with an enhancement of $\sim 10$ over the quiescent count rate (Ambruster et al. 1994) to large events that can last up to 24 hours (Sciortino et al. 1999) and peak at 300 times the quiescent count rate (Favata et al. 2000).

\section{Chandra Observations}

The Chandra X-ray Observatory observed EV Lac for 100 ks, starting 2001 Sept. 19.8 and ending 2001 Sept. 21. The High Energy Transmission Grating Spectrometer was in place to record high spectral resolution X-ray spectra. Initial data reduction used the CIAO "threads"; final steps made use of custom IDL procedures. Following order sorting and resolving photons into spectral orders, a light curve was made of all events falling within the spectral extraction window of the Medium Energy Grating (MEG; see Figure 1). This light curve revealed the presence of numerous low-amplitude flares, in addition to a few high contrast flares. There is approximately $40 \mathrm{ks}$ of quiescence at the beginning of the observation.

We investigated the high amplitude flares with smaller time binning. Two flares (Flare 2 and Flare 7 in Figure 1) exhibited fast time structures which were evident using temporal bins of 60 seconds. The total flare durations were $\sim 8$ minutes (Flare 2) and $\sim 25$ minutes. Flare 2 had a maximum enhancement of $\approx 15$ over the quiescent count rate, the largest enhancement of the X-ray flares. We detected $\approx 9$ flares, leading to a flaring rate of 0.32 flares $\mathrm{hr}^{-1}$. This is in agreement with Leto et al. (1997)'s B band flaring rate of 0.16-0.37 flares $\mathrm{hr}^{-1}$. 
We originally set out to perform a time-resolved spectroscopic investigation of the difference between quiescent and flare conditions in the corona of EV Lac. However, the frequency and timescales of the flares made it impossible to extract spectra from individual flares (the small amount of quiescent time also hindered examination of differences between quiescent and flare conditions). As a first step in investigating flare-related spectral changes, we categorized the flares as either "large" in amplitude (Flares 1, 2, 7, and 8), or "small" in amplitude (Flares 3, $4,5,6,9)$, and extracted three dispersed spectra, corresponding to Quiescence, Large Flares, and Small Flares. The continuum level for the Large Flares was $\approx 710^{-13} \mathrm{erg} \mathrm{cm}^{-2} \mathrm{~s}^{-1} \AA^{-1}$, an enhancement of $\sim 3$ over the Quiescence and Small Flares. The continuum at $\lambda \leq 10 \AA$ is formed predominantly by high temperature plasma ( $\mathrm{T}>30 \mathrm{MK})$, and this enhancement signals an increase in the amount of high temperature plasma present during the Large Flares.

The quality of the dispersed spectra was not sufficient to perform a selfconsistent differential emission measure (DEM) distribution analysis and accompanying abundance analysis; however, we were able to make use of line ratios to examine relative abundances. We used lines which have significant temperature overlap to determine if there were any variations in the abundances between quiescent and flare conditions. For Si XIII $\lambda 6.65 / \mathrm{Mg}$ XII $\lambda 8.42$, $\mathrm{Mg}$ XI $\lambda 9.17 / \mathrm{Ne} \mathrm{X} \lambda 12.13$, and $\mathrm{Ne}$ IX $\lambda 13.45 / \mathrm{O}$ VIII $\lambda 18.97$, there was no evidence of enhancements in the abundance ratios between quiescence and flares.

An initial DEM analysis was done of the entire observation, neglecting any activity variations. The results (based on the procedure described in Osten et al. 2003) confirm previous investigations of the coronae of active stars: an enhancement in the DEM around 8-10 MK, electron densities $\mathrm{n}_{e} 10^{11}-10^{13} \mathrm{~cm}^{-3}$, and an abundance pattern varying inversely with first ionization potential. A more complete DEM analysis utilizing both X-ray and UV spectra will be done, in a separate (longer) paper.

\section{VLA Results}

EV Lac had been observed with the VLA on several occasions prior to the current campaign, but never in a systematic fashion to study flares. Typical quiescent flux densities are $\approx 0.6$ mJy on 2001 September 19 , and on 20 September preceding the large enhancement. Our continuous observations at $\mathrm{X}$ and $\mathrm{C}$ bands ( 8 and $5 \mathrm{GHz}$, respectively) on 20 September were able to probe the nature of extreme radio flares: In the course of about 30 seconds, the flux density from EV Lac underwent an enhancement of approximately 100, reaching a peak of 60 mJy at X-band $(3.6 \mathrm{~cm}$; see Figure 1$)$. The flare is evident at both frequencies, and shows a smooth decay which is indicative of well-trapped electrons. Examination of the evolution of spectral indices $\left(\mathrm{S}_{\nu} \propto \nu^{\alpha} ; \alpha \sim 1.5\right.$ initially) show an initially fast decline of $\alpha$, then a flattening at about 1 hour from the flare peak. This is indicative of a change from optically thick conditions during the flare rise and peak, to optically thin conditions at the late stages of the flare decay.

\section{Multi-Wavelength Comparisons}

Figure 1 shows the overlapping observations from the optical $U$ band, X-ray, and $3.6 \mathrm{~cm}$ radio datasets. The large radio flare is accompanied by a large $\mathrm{U}$ 
band enhancement of $\approx 3.3$ magnitudes. The optical photometric enhancement has no accompanying increase in optical chromospheric transitions. There are numerous flares occurring during this time in the X-ray, which makes it difficult to identify any one X-ray flare as the accompanying flare for the optical/radio flare. The impulsive X-ray flare with a maximum enhancement of $\approx 15$ noted in $\S 2$ appears to precede the optical/radio flare by $\sim 1200$ seconds. If one is looking for multi-wavelength patterns as seen in the Neupert effect, then the very small enhancement (Flare 3 in Figure 1) must accompany the large optical/radio flare with a time delay of $\sim 2600$ seconds. If this is the case, then Flares 1 and 2 are notably deficient in the optical/radio emission.

The UV and X-ray data, which overlapped from 2001 September 20.7-20.9, show a general agreement: two small UV flares occur during the rise phase of two moderate X-ray flares. Under the framework of the Neupert effect for interpreting stellar flares, the ultraviolet measurements represent the result of nonthermal heating (coming from particles which presumably would produce radio gyrosynchrotron emission), and the increase in X-ray intensity occurs as a direct result of the nonthermal heat input, producing thermal X-ray emission.

\section{Discussion \& Conclusions}

The Neupert effect from multi-wavelength studies of the solar corona (Dennis \& Schwartz 1989) has been invoked to explain observed temporal trends in multiwavelength observations of stellar flares (e.g., Güdel et al. 1996). At its heart the Neupert effect is an observational statement that optical, radio, or UV flares show an enhancement which precedes thermal coronal (extreme ultraviolet or soft X-ray) flares. The interpretation is that the (optical, radio, UV) emission is indicative of some kind of nonthermal heating occurring during the flare. This nonthermal energy input into the stellar atmosphere results in a radiative instability which is responsible for the transient heating of chromospheric material to coronal temperatures, producing $\mathrm{X}$-ray radiation.

The optical/radio flare on EV Lac is in sharp disagreement with the Neupert effect interpretation of flares on stellar coronae. What is this flare telling us? Radio enhancements signify that nonthermal particles are present in abundance; a conservative estimate of the average particle energy (using a flux density of 60 $\mathrm{mJy}$ and a source size of the stellar diameter to determine brightness temperature $\mathrm{T}_{b}$ ) leads to $<k T_{b}>\sim 0.5 \mathrm{MeV}$. The U-band flare indicates that heating of the chromosphere is taking place, although not in the chromospheric lines. And the lack of corresponding X-ray emission signals a disconnect between the nonthermal energy input and coronal heating. This could be indicating a special site for the flare, where atmospheric column densities prevent the traditional flare heating scenario from taking place, instead confining heating to temperatures of less than $10^{4} \mathrm{~K}$. This idea awaits more rigorous testing.

\section{References}

Ambruster, C. W., et al. 1994, BAAS, 26, 866

Caillault, J.-P., Drake, S., \& Florkowski, D., 1988, AJ, 95, 887 
Delfosse, X., Forveille, T., Perrier, C., \& Mayor, M. 1998 A\&A, 331, 581

Dennis, B. R., \& Schwartz, R. A. 1989, Sol. Phys., 121, 75

Güdel, M., Benz, A. O., Schmitt, J. H. M. M., \& Skinner, S. L. 1996 ApJ, 471, 1002

Favata, F., Reale, F., Micela, G., Sciortino, S., Maggio, A., \& Matsuomoto, H. 2000, A\&A, 353, 9987

Johns-Krull, C. M., \& Valenti, J. 1996, ApJ, 459, L95

Kodaira, K., Ichimura, K., \& Nishimura, S. 1976, PASJ, 28, 665

Leto, G., Pagano, I., Buemi, C. S., \& Rodonò, M., 1997 A\&A, 327, 1114

Osten, R.A., Ayres, T. R., Brown, A., Linsky, J. L., and Krishnamurthi, A., 2003 ApJ 582, 1073

Saar, S. H. 1994 IAU Symp. 154 Infrared Solar Physics, ed. D. M. Rabin et al. Sciortino, S., Maggio, A., Favata, F., \& Orlando, S., 1999, A\&A, 342, 502

White, S. M., Jackson, P. D., \& Kundu, M. R., 1989, ApJS, 71, 895
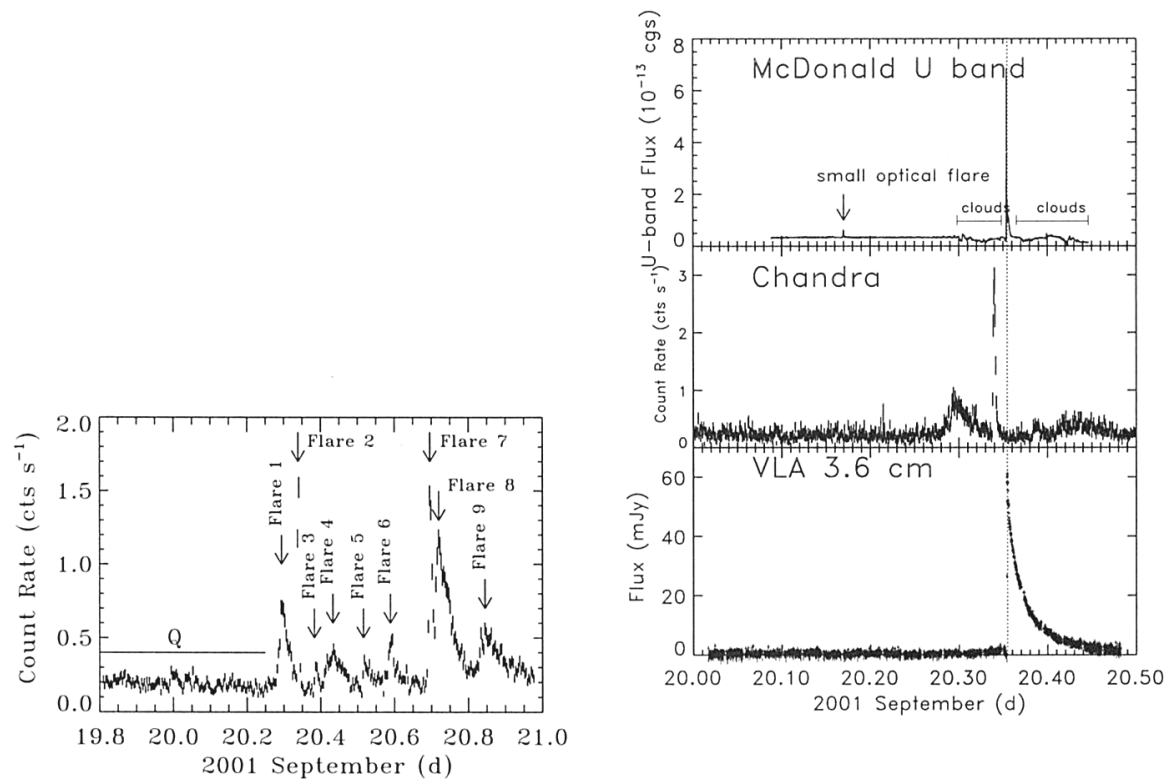

Figure 1. (left):Light curve of MEG X-ray photons throughout the 100 kilosecond Chandra observation. Each tick mark represents a duration of 300 seconds. (right): Illustration of optical (top), X-ray (middle) and radio (bottom) multi-wavelength correlations. Optical time binning is 3 seconds; X-ray light curve bins are 60 seconds, and VLA $3.6 \mathrm{~cm}$ light curve bins are 10 seconds. Large optical and radio flares appear simultaneously, and there is no apparent correlation with $\mathrm{X}$-ray variations. 